when he had an attack of jaundice. He recovered from this, and remained well till, four years ago, he became subject to bilious attacks accompanied by irregular action of the bowels. Three years ago, after much annoyance and mental excitement, he suffered from carbuncles on his back. Since that time he had been in bad health; suffering from a feeling of numbness in the left leg, and occasional severe attacks of diarrhoa. He complained of occasional neuralgic pains, and at times entire absence of sensation in the left foot. He walked into my room like a man debilitated by disease, but in no way resembling a paralytic. He complained of having been affected with seminal emissions. I examined him very carefully afterwards in bed, and could find no trace of loss of parallelism or loss of motor power. I could not satisfy myself, by the æsthesiometer, of any material impairment of tactile sensibility; no tenderness of the spine and no abdominal derangement were traceable; nor was there any disease of the rectum or prostate. I commenced the treatment with steel, and the application of an anodyne liniment; and ordered wine, beer, and nutritious food. The patient, being a teetotaller, was with great difficulty persuaded to take any fermented liquors. I obtained a specimen of the morning and evening urine before my next interview, when I had a consultation with a leading physician on the case. Both specimens had a specific gravity of 1035 , were strongly acid, exhibited no increase of phosphates, and contained neither sugar nor albumen. There were no crystalline forms in either, and specifically no oxalates; but both specimens almost solidified on the addition of equal volumes of nitric acid, by the formation of nitrate of urea. The joint examination elicited no proof of any organic disease; and it was agreed that the case was one of azoturia. The quantity of urine never was large that was passed in the twenty-four hours; it rarely attained to three pints. I find that on one occasion I had the advantage of obtaining a volumetric analysis from Dr. Ringer; and the estimate was seven drachms and a half in the twentyfour hours-an amount considerably above what Dr. Parkes considers the average in the healthy adult. The gentleman remained under my treatment for three weeks; and improved materially under the administration of mineral acids, opiates, and tonics of various kinds. It was not till after twelve days' observation that I discovered a few oxalates in his urine. In a medical report which I gave the patient at his request, on leaving town, I stated that I regarded the azoturia as the source of debility; and that the drain caused by a persistent waste of tissue, indicated by an excess of urea is the urine, sufficiently accounted for the anomalous nerve-symptoms to which he was liable.

I saw the same gentleman again at the end of 1862, when he was passing through London after he had, without medical advice, been taking the waters of Kissingen, and thereby brought on severe diarrhœa. He was naturally weak in consequence; but the urine presented a specific gravity of 1025-lower than I had ever seen it formerly, and I have a memorandum that it contained but little urea.

There are many insidious morbid conditions for which we may find an adequate explanation in the urinary secretion, with important hints as to treatment and regime. The insidiousness of degenerative renal disease as indicated by albumen, is a point that can scarcely be too often mooted; and it is surprising how often and how long patients continue ailing and complaining of feebleness and want of power without presenting any very tangible symptoms, when an examination of the urine reveals the cause of the anæmia, the weakness, and the apnœa, and at once suggests the proper indication. Although albuminuria is a much more frequent cause of these insidious symptoms than azoturia, still I am satisfied that there are numerous cases of chronic disease going through a weary life, for which the undue waste of the nitrogenous tissues indicated by a high specific gravity of the urine and an excessive proportion of its urea affords a satisfactory explanation.

\section{ON SYPHILITIC ULCERATION OF THE PALPEBRAL CONJUNCTIVA.}

By JoHn Windsor, Esq., Consulting Surgeon of the Manchester Eye Hospital.

OF the secondary symptoms of syphilis, there is one which appears to have been rarely observed by writers on this subject, or, if observed at all, to have scarcely attracted particular notice or description : I allude to an ulcerated state, evidently, from its history, of syphilitic origin, appearing on the palpebral conjunctiva, and generally involving the adjoining tarsal cartilage. It is certainly an affection, as compared with other secondary symptoms, of rather rare occurrence; and in my own practice, of now a long duration, I do not remember (excluding, perhaps, two or three instances of it in congenital syphilis) to have met with more than about seven or eight cases; and of these, two of which occurred recently, I am enabled to add a brief report. Before doing so, however, I wish to premise a few remarks.

The subject of syphilis has for a long time, but more especially at a comparatively recent period, attracted considerable attention and research, both in this country and abroad; from which, I believe, a more correct knowledge of the specific distinctions of the disease has been acquired than was previously possessed.

At the present time, the conclusion arrived at seems to be, that there are two forms of primary syphilitic ulceration. One is the soft suppurating sore, sometimes accompanied, or soon succeeded, by enlargement, and not unfrequently suppuration, of the adjoining inguinal glands, but not contaminating the system, although communicable to others, and capable of reproducing repeatedly, by inoculation, similar sores in the same or in a different individual. It may be observed that occasionally, from some inflammation being excited, a degree of hardness is produced even in this form, but still distinct from the circumscribed and generally characteristic induration of the other.

The second form is the indurated sore, sometimes called the Hunterian chancre, the character of which is, in the first place, the secretion of an adhesive material, fibrine instead of pus (which latter occurs in the other form) ; thus appearing at first often rather as a pimple or hard tubercle, than a pustule. With this second form there is also not unfrequently some indurated enlargement of the adjoining inguinal glands, but it is not apt to undergo a suppurative process. This second form is the one which infects the blood; enters into the patient's system, producing secondary symptoms; and, although communicable to others, is not autoinoculable, except in its earliest period, before the adhesive effusion takes place. It, as a rule, occurs only once during life; whilst the first form may occur many times.

The first form I would compare to the porrigo (impetigo) larvalis (Willan), which, by contact, is often conveyed from one part of a child to other parts, or to those of its nurse. The second form I would rather compare, as infecting the general constitution, and mostly occurring once only during life, to small-pox, although 
differing by the virus of the latter being in a great degree speedily eliminated from the system. Like small-pox, also, it has a period of three to seven weeks, or more (according to Mr. Henry Lee), of incubation, before the characteristic circumscribed hardness appears in the original fissure, abrasion, or pimple; and we know that another period intervenes before the appearance of secondary symptoms.

It seems possible that these two forms of syphilitic ulceration may coexist in the same person, and thus be communicated simultaneously to another; and thus, probably, the appearance of a pustule, not attended by a period of incubation, may sometimes precede the development of the true incubated chancre.

The first, or soft suppurating sore, is curable without the use of mercury, and its specific character may be generally effaced by the early application of caustics. The second, or indurated infecting sore, along with its secondary constitutional symptoms, or, as not unfrequently occurs, in its congenital hereditary form, yields to the action of this valuable medicine, administered seasonably, moderately, and cautiously, according to the circumstances or peculiarities of each case. For our present more precise knowledge of syphilis, we are especially indebted to the researches, experimental and practical, of Mr. Henry Lee, as shown in his recent work on Syphilis; although, doubtless, there have been other able coadjutors, both domestic and foreign, in the investigation of this important subject.

With regard to the more immediate subject of this paper, it seems, as far as I know, that Mr. Lawrence was the first to give an accurate description of it, as published in his work on Diseases of the Eye, as also in his work on the venereal disease of that organ. It has been since mentioned in the works of Mackenzie, and also of Middlemore, on the eye; and in the manual of Wharton Jones; perhaps, also by others ; but I do not see it mentioned in 'Travers's excellent Synopsis, nor in Morgan's Lectures on the Eye. Neither have I seen any account of it in Scarpa's or Weller's works on the diseases of the eye. It is adverted to in Desmarres's, and also in Déval's, Traités des Maladies des Yeux; but not in the earlier works of Demours or Desmonceux; nor is it noticed, I think, except very slightly, by Carmichael of Dublin, or Sir Charles Bell; nor in the comprehensive work of Langston Parker (third edition) on Syphilitic Diseases; yet Mr. Parker notices particularly the frequent occurrence, in secondary syphilis, of inflammation and ulceration of the mucous membrane investing the nares.

Some of the first examples of the affection which I had an opportunity of seeing occurred in persons whose cases I have recorded as instances of the phagedænic form of syphilitic ulceration; and the sores appearing on the palpebral conjunctiva are noticed incidentally, as seen in a subsequent period of the progress of the disease. Indeed, at that time (1821) my attention had not been particularly directed to the subject; and the first edition of Mr. Lawrence's work, containing his remarks on it, was not published until 1833 .

CASE I. I was called on May 16th, 1825, to a gentleman named $R$. Allen, on account of a rheumatic affection. At the same time, a hard tumour, of about the size of a pea, presented itself near the frænum penis, which gradually became more inflamed, and afterwards ulcerated; the ulceration soon extending much over the part between the prepuce and the glans, forming a very large irritable sore, with hard ulcerating edges. In this state it proceeded for a few weeks; and then, after the continued use of decoction of sarsaparilla, aperients, and milk diet, with rest, it began to assume a healing aspect, and was nearly well, when he irritated it by exposing himself to a fresh infection; but, by persevering in the same treatment, it again presented a healthy healing appearance, under the application of poultices and mildly stimulating mercurial ointments. On August 12th, it was nearly well; and an eruption on the forehead, which had troubled him for about six weeks, had also disappeared, excepting the marks, under the same treatment. Recently, however, the disease had reappeared in the form of five or six blotches on the forehead, of a circular form, of about the size of a sixpence, or smaller, flat in the centre and elevated at their margins, all of a coppery red colour; one on the head, amongst the hair, discharged matter occasionally from under a crust; and one on his forehead was nearly in the same state. There were two or three smaller eruptions on his face, in the form of acne or ecthyma. He continued to take decoction of sarsaparilla ; and a solution of hydrargyrum corrosivum sublimatum was applied. Oct. 1st. The eruption on the head and face was nearly well. For the last fortnight he had complained of his throat, which, on inspection, looked rather red, but was not ulcerated. The sore on the inferior part of the glans, or rather on the corpus spongiosum behind it, continued open, but had a clean healthy appearance.

Nov. 12th. He called upon me. Having gone out much during wet cold weather, and also living rather irregularly, he was not so well; his throat had become ulcerated on both tonsils, and on the posterior part of the pharynx, with hoarseness and some cough. The eruption on the skin continued better; the sore on the penis about the same. His pulse was accelerated; his appetite and general health indifferent. I directed him to stay at home, and to live much on milk diet; prescribing him pills twice a day of extract of conium and rhubarb (sing. gr. iij); and decoction of sarsaparilla with dilute nitric acid after each dose; also to use frequently a gargle of oxymuriate of mercury with tincture of myrrh and rose-water.

Nov. 19th. A sore (serpiginous), which he had had on his forearm, was now healing from the centre, whilst its margin was yet rather ulcerating. The sore on the penis was healing in some parts from its edges, but in others was rather ulcerating. His throat was about the same. A very large abscess, formed in the perinæum in consequence of a blow on the part, discharged freely. A few scattered spots appeared on the trunk, but were scarcely ulcerating.

Nov. 27th. He was in nearly the same state. The phagedænic or serpiginous sores were still rather extending. The tonsils and posterior part of the pharynx were almost covered with ulcers. One also, for the last ten days, had formed under the superior palpebra of the left eye. That on the penis was extending at the margin, although healing at its middle. That on the arm was cicatrised, but was red, hard, and elevated. There were several sores on his body, but there was scarcely any discharge from them. The sore from the blow in perineo seemed healthy, but was still large. The sores on his body were dressed with resin cerate, and those in the throat were touched once or twice a day with oxymel æruginis. The other medicines were continued.

Dec. 18th. The oxymel æruginis, with an equal quantity of water, the parts being washed immediately afterwards, seemed to have had a good effect on the sores of the throat, penis, forearm, and conjunctiva palpebræ; and all of them appeared healing, as was also slowly that of the perinæum. The pulse was still frequent, and the appetite indifferent.

Dec. 28th. The sore on the penis, after being open 559 
nine months, was now just healed. The throat and other parts were also rather better, and his general health was a little improved.

Jan. 1st, 1822. About a dozen scabbed sores existed on the back and right side of the trunk, varying in size from that of a sixpence to a shilling. I prescribed him hydrargyrum cum cretâ and rhubarb (sing. gr. iij) three times a day, with an antimonial demulcent mixture.

Jan. 30th. He was nearly in the same state. Another small ulcer, which had appeared under the same palpebra, but rather nearer the external canthus, was touched with the oxymel æruginis.

Feb. 18th. The conjunctival lining of the superior palpebra was nearly healed, but appeared of an unequal white and red colour, and was not perfectly smooth. The throat also appeared rough and ulcerated in points. Small ulcers on the penis alternately healed and broke out again. The incrustations on the trunk were in general falling off, but some formed again. His general health seemed rather improved.

April 20th. The state of the throat, conjunctiva, penis, and perinæum was about the same; and the cutaneous incrustations continued in the same state. He had some cough, and a little œdema of the lower extremities towards night.

June 4th. The palpebral conjunctiva continued a little red and rugose, but not ulcerated; the other parts were much the same. The cutaneous scabs had in a great measure fallen off. The cough and œdema of legs were nearly gone. Pulse frequent; appetite and sleep moderate. He walked out now a little almost daily.

June 21st. The palpebral conjunctiva of the left eye was again ulcerated; that of the right one had also latterly become affected, first by a few enlarged vessels appearing, which soon increased so as to produce a dense red spot, the precursor, probably, of ulceration. Numerous scattered spots of acne or ecthyma, with a yellowish suppurating point, had appeared nearly all over the body, producing much soreness. The throat was also in the same ulcerated state as it had been occasionally before. For the last four or five weeks he had been using, by his own desire, a mild mercurial course ; but during this time there had been rather an aggravation of his complaint.

July 15th. The eruption was rather worse than better, affecting the face (especially the nose, which was almost covered with scabs and ulcers), the head, back, arms, and indeed the whole surface of the body. Some of the ulcers were covered with large crusts; others were discharging, and requiring to be dressed. The throat, palpebral conjunctiva, perinæum, and penis were much in the same state as before. I desired the mercury, which had been taken lately in very minute doses, to be entirely omitted; and ordered him to take only a mild aperient pill, and the decoction with acid as before.

Oct. 30th. Since last report, he had been staying in Liverpool ; and during that time had been chiefly under the care of Dr. Macartney, who prescribed for him compound decoction of sarsaparilla, with an addition of extract of sarsaparilla, three tablespoonfuls three times a day; one-eighth of a grain of bichloride of mercury three times a day; and six minims of liquor arsenicalis three times a day; also antimonial powder, with extract of conium and opium, each night; and black oxide of mercury for fumigation. $\mathrm{He}$ also took twice a day a pill of two-thirds of a grain of black oxide of mercury, with ten grains of compound chalk powder and a grain of opium. The warm sea-bath was tried, but it seemed rather to increase the eruption. At present he was much better. The scabs had in a great measure fallen off, leaving 560 the parts healed. The ulcers of the conjunctiva were healed; the penis also. The throat was better, and the perinæum was healing. His general health was improved. Pulse natural ; appetite moderate.

Dec. 6th. The pills and arsenical drops had been continued; but, some diarrhœa having come on, I desired him to discontinue them for a short time. A spot of ulceration, of about the size of a split pea, had appeared on the velum pendulum palati. $\mathrm{He}$ was prescribed half a pint of compound decoction of sarsaparilla twice daily, and to use the gargle as before for his mouth and throat.

Feb. 20th, 1823. About three weeks since, whilst there was a very severe frost, he continued to go out daily. Calling upon him at this time, I found the throat, tonsils, uvula, and pharynx presenting a sloughy appearance. Two sores upon the front of his leg had also a nearly similar aspect. His pulse was frequent, but his appetite tolerable. I desired him to keep the house, to live pretty well, to use his gargle warm, and to apply to his leg a linseed poultice. In about a week, the throat became cleaner; the greater portion of the uvula, sloughing off, came away. The ulcers on his leg had a sloughy and ulcerating appearance. I desired the linseed poultice to be exchanged for a bread and milk one, which immediately effected a favourable change, the sores assuming a granulating state, and soon beginning to cicatrise. His general health again improved. The conjunctival sores healed; those of the penis and perinæum nearly so. His skin was pretty free from tuberculous or ecthymatous eruption, but almost covered with large cicatrices.

March 27th. He continued better as to the external complaints, these being confined to a few small clean ulcers on his legs; but he had now become decidedly the subject of ascites, with œdema of the lower extremities.

Having already related this case so much at length, I will refrain from further details, only stating that, by the means adopted, the dropsical symptoms were removed.

March 1825. After the last report, he had a return of tuberculous or acne-like eruption all over his body, with much headache and some sore-throat; but he had now for some time been in tolerable health, only he was considerably altered in his appearance by the very long duration of his illness and the numerous cicatrices on his face.

Since reading the work of Langston Parker, I am inclined to think that the disease would probably have had its course arrested by larger use of some mercurial fumigation.

Having dwelt so long on the recital of the above, as I consider, very interesting case, I will endeavour to be more brief in what has to follow.

CASE II. In October 1827, I saw a gentleman named Daniels, affected much in the same way as Allen was. He had ulcers on the palpebral conjunctiva and tarsal or ciliary margin; also an enlargement of the left testis, or what has been called syphilitic arcocele. Under the use of decoct. sarzæ and liquor arsenicalis, he soon improved, and in about two months was almost well, the sores being all healed, except one on the left arm, now also healing. The testis also was much reduced in size, under the application of ol. palmæ camphor. I saw him occasionally for a while; and on April 5th, 1828, I found he had been well for some time. I should add, that he had been under good medical advice for some time before I saw him.

CASE IIr. September 1841. I attended a Mrs. B., affected with an eruption of rupia and the phagedænic form of secondary syphilis. The throat was ulcerated, and there was ulceration of the palpebral

7

宁 
conjunctiva near the ciliary margin. There was a large prominent crust in the hairy scalp, near the forehead; and a smaller one behind it. There was an ulcer on one thigh, succeeded by a few others on the foot. She had a good deal of pain, with some swelling and tenderness of the knees.

She was soon relieved by the use of anodynes and the iodide of potassium. The throat healed over very soon; and also the ulcer on the thigh, which was dressed with resin cerate and a small addition of red precipitate. The ulceration of her palpebræ continued for some time; and the crusts on the head were very slow in falling off and healing, the larger ones especially.

CASE Iv. Ann Barlow, aged 23, married about fourteen months, came under my care as an outpatient of the Manchester Eye Hospital October 28th, 1847. The ciliary marrin of the superior palpebra of the right eye was ulcerated away, except a small portion towards the external canthus. There was also an ulcerated state in the middle portion of the ciliary margin of the lower palpebra, which was more especially covered with a puriform fluid. The globe itself was unaffected, only it had been a little red occasionally. There was an enlarged gland, rather sore when touched, in front of the right ear; and a similar one just below the angle of the inferior maxilla on the right side. She complained of much pain, of a burning, throbbing, and lancinating kind, in the palpebræ and surrounding parts. That side of her face was hotter and redder generally than the other. About fourteen weeks previously, a small ulcer appeared under the upper eyelid. Her medical attendant at Ashton-under-Lyne applied to it stimulants and escharotics; but the ulceration extended to the tarsal margin, and had extended during the last fortnight. Pulse 96; tongue pretty clean; bowels confined; appetite indifferent; she complained of headache; the catamenia had not appeared since her eye was affected; and she thought she might be pregnant. She had had one child born at seven months-on May 3rd. It died of convulsions, when five weeks old. It had no particular eruption on the skin. About seven months after marriage, she became affected with sore places about the pudenda; and, some time before that, she had much vaginal soreness. Some months afterwards, her throat became sore and ulcerated ; it was about two months in healing. Afterwards, her eye became affected; and, within the last fortnight, red hard spots had appeared on her legs; they were very sore to the touch, and apparently intermediate between nodes and erythema nodosum. There were several of them on the right leg, and a few on the left; they were not all on the periosteum. There were about three also on the left thigh, quite cutaneous. She could ascribe no cause for the complaint, except infection from her husband, who appeared to have had syphilis. Previously. to her marriage, she was very healthy.

A colleague of mine, who accidentally saw the case, was, at first view of the palpebræ, impressed with the idea that it was a malignant and intractable affection; but, judging from its history and my former experience, I was inclined to entertain a more favourable opinion. I prescribed ten grains of blue pill three times a day; and with each dose two tablespoonfuls of an iodide of potassium mixture, and zinc ointment to the tarsi. 亏ेxij.

Bo Potass. iodid. Эij ; syrup. pap. f. $j^{\mathrm{iv}}$; aquæ ad f.

Nor. 1st. The ulceration of the palpebræ looked cleaner, more kindly, and was attended with less pain.

Dec. 1st. The ulcerated parts were now nearly healed, the improvement having progressed regularly since last report. The tarsal margins were again nearly even, being only slightly indented in one or two points. She had continued her medicines regularly. Sulphate of copper had been occasionally applied to the ciliary margin.

Jan. 10th, 1848. She continued pretty well, and scarcely any difference was observable in the two eyes.

September. She called again to express her thanks, and to show that her eyes remained well.

CASE v. July 29th, 1840. Jane Beresford, aged 21 , married, came under my care as an out-patient of the Manchester Eye Hospital. She said that, about twelve months since, she became infected with syphilis by her husband. After suffering from secondary symptoms, in a few weeks she became affected with sore-throat, followed by ulcerating blotches on her legs and back, not more than two or three on the former, and one or two on the latter, all now nearly healed. Latterly the palpebræ had become affected, presenting a reddish swollen appearance, along with some ulceration of the ciliary margin, extending a little inwards upon the conjunctiva. I prescribed for her the same treatment as mentioned in the preceding case.

Aug. 3rd. The palpebræ were still reddish, and a little swollen; but the ulceration was already almost healed, and her general health was improving.

Nov. 12th. She had been for some time pretty well, and discontinued her medicines soon after last report.

CaSe vi. On December 30th, 1863, I was consulted by Mr. N., a middle-aged man, unmarried, on account of an affection of his eyes and throat, which had been coming on for a week or more. The ciliary margins appeared red and somewhat swollen; and, on everting the superior palpebræ, two small ulcers were visible on one of them, and one only on the other. Each ulcer was a little excavated, presenting a yellowish base, and was about the size of a split pea. They were all on the palpebral conjunctiva, quite within the ciliary margin, and, therefore, not observable until the palpebræ were everted. On examining the throat, a rather large yellowish ulcer was seen on the left tonsil. I prescribed him the same medicines as in the preceding cases, and a lotion for his eyes.

Jan. 3rd, 1864. The ulcers on the palpebral conjunctiva and left tonsil had lost their yellowish coating, and seemed healing.

Jan. 9th. The ulcers were now scarcely visible either on the palpebræ or tonsil. The medicines were continued.

Jan. 15th. The ulcers were now healed, and his general health was improved. He had, however, still some ulcers on his legs-a frequent, if not general, concomitant of this form of syphilis; the ulceration being often of a sloughy, phagedænic, or serpiginous character, at least in one period of its progress.

CASE vir. October 29th, 1864. I was consulted by a Mr. B., aged about 30 , married, residing until recently at New York. He had an ulcer occupying the tarsal margin of the left superior palpebra, and extending somewhat within the conjunctival surface. The surrounding textures appeared reddish and thickened. He first noticed the affection about three weeks ago, and it had gradually become a little worse. He had not hitherto used any particular treatment for it. About eighteen months since, he became affected with a cutaneous eruption in large spots (ecthyma?) on his legs and shoulders, and also with ulcerated sore-throat; but these were now all healed, leaving rather large cicatrices on the skin. He said that he had gonorrhca about the commencement of 561 
his complaint, but was not certain as to the existence of primary sores. I prescribed for him nearly the same medicines as in the preceding cases; the blue pill being united with an equal quantity of extract of conium and mixture of iodide of potassium. The affected lid to be brushed occasionally with tepid water, and anointed afterwards with the milder mercurial ointment.

Nov. 2nd. The ulceration of the ciliary margin and adjoining palpebral conjunctiva, as well as the accompanying redness and swelling, were diminished. The cicatrices on his legs, etc., varied in size from that of a sixpence to about that of a shilling.

Nov. 7th. The external ulceration of the tarsus was now healed; that of the conjunctiva internally was contracting, and presented a healthy granulating surface. The swelling and redness of the margin had disappeared.

Nov. 19th. The ulceration was quite healed, leaving a slight, scarcely perceptible depression in the site of the lately ulcerated.ciliary margin; and the cilia corresponding to that part had quite disappeared.

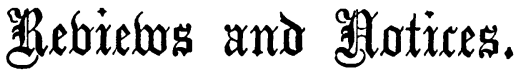

Report on the Cheap Wines from France,

Italy, Austria, Greece, and Hungary, etc. By Robert Druitt, M.R.C.P., etc. Pp. 179. London: 1865 .

THE writer advocates the largely increased use of wine in diet and in medicine, and the use of greater variety. His is not a formal treatise on wine; but he takes the various cheap wines actually on sale in London at the present moment, and describes their qualities and uses. First, he discusses the difference between pure wine and fortified wine, observing that the English differ from every other civilised people on the face of the earth by their predilection for wine which has been dosed with about 20 per cent. of proof spirit. He shows that common spirit is more or less contaminated with fusel oil and other irritating substances, and is made out of the cheapest and commonest grain; and that this common spirit is largely exported to Portugal, and returns in the shape of port wine! He shows that pure wine, of natural strength and unfortified, has about 20 per cent. of natural spirit, derived from the fermentation of its saccharine element. He teaches us how to judge of wine, laying especial stress on the body of wine-i.e., the totality of its sapid and aromatic ingredients, as contradistinguished from mere alcoholic strength. A mixture of spirit and water, he says, however strong, has no body. The question of acidity is also treated of, and a table is given with new determinations of the acidity of twenty-four kinds of wine. 'The headaches, heartburn, gout, etc., which follow the use of wine, he attributes to the wine being imperfectly fermented, or to the admixture with it of ardent spirits; and, let the wine only be pure, and there is no fear in drinking of it plentifully. Taking half-a-crown per bottle as a definition of cheapness, he gives a description of more than a hundred samples of cheap wine of all sorts which he has purchased and drank in London within the last two or three years; beginning with the Bordeaux and Burgundy; then taking the less known Greek, Hungarian, Austrian, and Italian wines, of which he details the names, prices, taste, alcoholic strength, etc. Amongst the Hungarian and Austrian, he describes all, whether cheap or dear. There are also remarks on cider, mead, champagne and sparkling wines, and on cheap port and sherry, etc. 'The whole is interspersed with constant eulogies of that mode of philosophising which the writer calls "empirical", and which consists in arguing from the results of experience, and not from $a$ priori considerations. Some space is devoted to an examination of hypotheses on which certain wines have been recommended by well known chemists and physicians, as, for example, liebig. He and some of his disciples have asserted that good wine is "rich in phosphorus"; and have asserted of some wines, that they are good because rich in phosphorus, which is a very different thing. Our readers must often have noticed a wine advertisement in which the words "No Life without Phosphorus" appeared in staring letters. It seems to give the writer a peculiar satisfaction to tear these hypotheses to rags and tatters, and to decry all systems of medicine and diet which do not rest upon experience.

The subject is naturally an attractive one even to teetotallers, and our author has certainly treated it in a practical and attractive manner. The British consumer of wine will derive both pleasure and profit from its perusal; but that he will be seduced from his strong and old-fashioned glass of port and sherry to the lighter draughts of Rhenish and Austrian, etc., produce, we will not venture to prophesy. Nevertheless, the author deserves our thanks for throwing his stone at the system of brandying natural wines and poisoning them with bad spirits.

A Manual of the Domestic Practice of Medicine. By W. B. Kesteven, F.R.C.S. Pp. 339. London : 1865 .

"IT is not," says the author, "the intention of this Manual to supersede the office of the professional attendant." But many persons are themselves obliged to practise medicine, because they cannot procure the benefits of medical skill; and it is for the benefit of such that this book is written. Mr. Kesteven says that he has reason to know that the first edition of his Manual has been of service both at home and in the colonies. We have no doubt it has been so; but then, unfortunately, books of this kind are apt to be a source of error to the well-intentioned. A few distinct practical rules, comprehensible to ordinary non-professional understandings, are no doubt very serviceable to all persons, as well to those who can as to those who cannot obtain professional assistance; but works which enter too minutely into the subject are likely to be the source of mischief in the hands of the good-natured busybodies who generally undertake the duties of doctor in village and outlying districts, where doctors' aid is not readily procured. How, for example, can my Lady Bountiful, even with the help of this manual, make a diagnosis of "Brain, Inflammation of ; Acute Water on the Brain; Acute Hydrocephalus"? Is it safe to tell that good lady that, in cases of this kind, she must give "an adult ten grains of calomel" and a senna purge ; and, after action of the bowels, "calomel in doses of two grains every four or six hours" until the gums become inflamed; and also to tell her that, "when this 